\title{
Quelques Grégarines Stylocéphalides parasites de Coléoptères Ténébrionides d'Egypte
}

\author{
Par Jean THÉODORIDÈS et Pierre JOLIVET
}

L'un de nous (P. J.) a eu l'occasion, lors d'un récent séjour en Egypte, de faire une centaine de préparations de Grégarines (frottis humides colorés au carmin acétique du contenu de l'intestin moyen de Coléoptères Ténébrionides) qui seront étudiées dans la présente note.

Ce matériel provient de la localité d'Abou Rawash (province de Giza), près des Pyramides, et a été récolté le 15 juin 1961 sous des détritus végétaux, sur le sable, au début de la zone du désert mais à proximité de cultures de melons et autres légumes.

Toutes les espèces observées appartiennent à la famille des Stylocephalidae Ellis 1912 strictement inféodée aux Coléoptères Ténébrionides (cf. Théodoridès et Pierre 1960).

\section{STYLOCEPHALUS LONGICOLLIS (Stein)}

Nous rapportons à cette espèce les Grégarines observées chez Blaps bifurcata Sol. en raison de l'aspect morphologique des céphalins et sporadins.

Un céphalin de $565 \mu$ environ avait un épimérite d'environ $140 \mu$ terminé par un gland allongé.

Les sporadins sont du type Stylocéphalide habituel. Blaps bifurcata est un hôte nouveau pour cette espèce que l'un de nous (Théodoridès $1955 a$ ) avait mise en évidence chez Blaps sulcata Cast. d'Israël.

\section{STYLOCEPHALUS EASTONI Théod.}

Nous avons retrouvé chez Blaps polychresta Forsk. d'Egypte cette Grégarine que l'un de nous (Théodoridès $1955 b, 1956)$ avait découverte et décrite chez Blaps inflata All. du Maroc.

Les individus provenant de ces deux pays ont une morphologie identique (cf. fig. 1).

Ils sont surtout représentés par des grands céphalins pouvant dépasser $1 \mathrm{~mm}$ de longueur et présentant un épimérite tout à fait caractéristique consistant en un mucron porté par une hampe (diamérite au sens de Filipponi 1949).

La longueur de l'ensemble diamérite + épimérite peut varier de $80 \mu$ à $170 \mu$ (dimensions intermédiaires $=100,125,130 \mu$ ) suivant les stades de croissance des céphalins et également suivant leur état de contraction ou rétraction. 

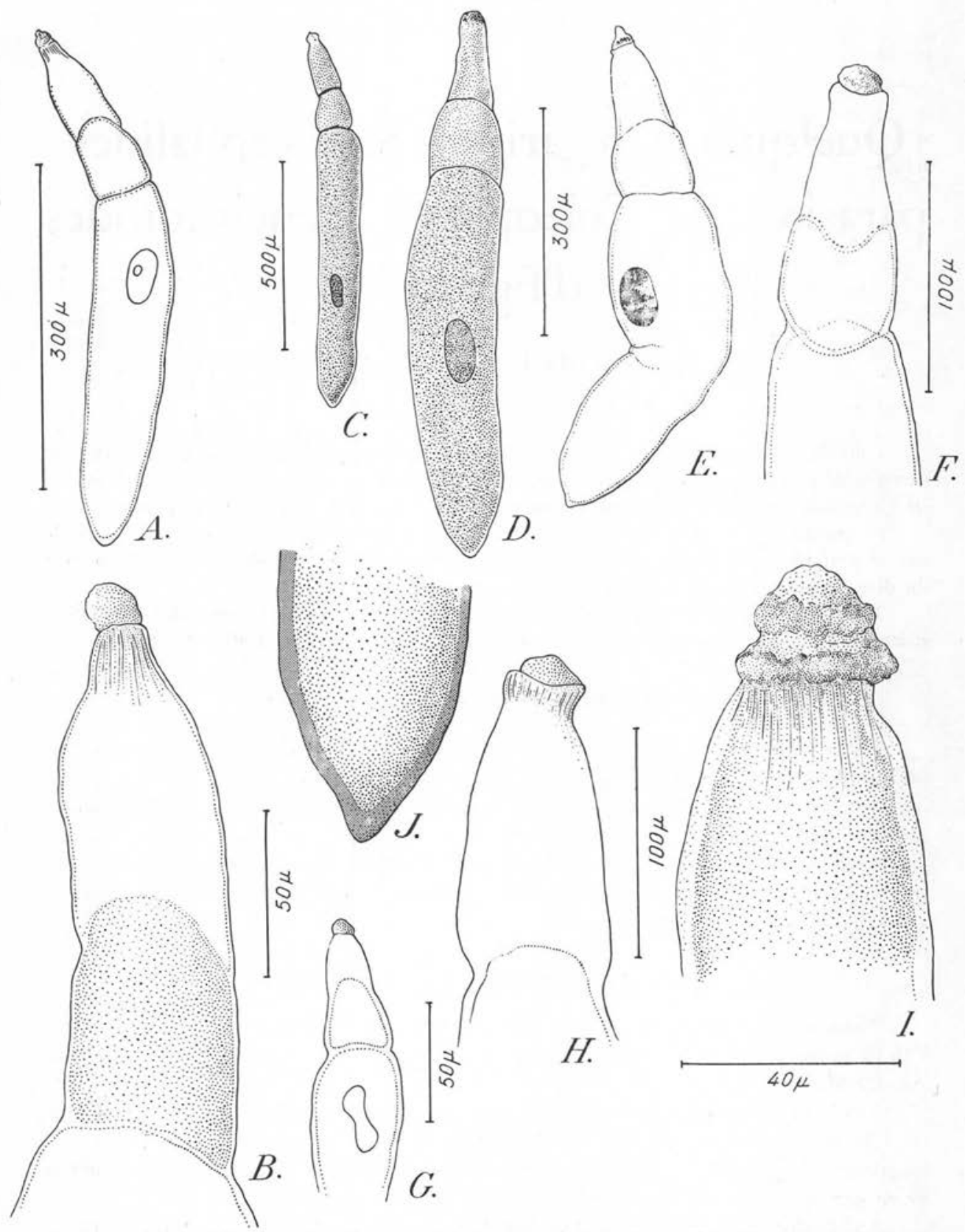

Fig. 1. - Stylocephalus eastoni Théod. A, céphalin observé chez Blaps inflata (Maroc). B, détail de l'épimérite du même. C, D, E, céphalins observés chez Blaps polychresta (Egypte). F, G, détail de la région antérieure. $\mathrm{H}, \mathrm{I}$, détail de l'épimérite. 
Comme nous l'avons déjà signalé dans notre description de cette espèce (Théodoridès 1956, p. 84), on distingue, en allant vers l'apex de l'épimérite, les trois zones suivantes: 1) zone finement ponctuée à la jonction du diamérite avec le protomérite (ces deux parties étant séparées par un fin septum) (fig. 1, A, B, F) ; 2) une zone de stries plus ou moins étendues (fig. 1, A, B, H, I) qui rebordent la gaine du mucron. Ces stries sont très nettes lorsqu'on observe les préparations avec l'objectif à immersion ; 3) le mucron proprement dit finement ponctué et d'aspect granuleux (cf. fig. 1, I).

Le protomérite est de forme quadrangulaire et la constriction au niveau du septum proto-deutomérite est peu indiquée.

Le deutomérite se termine généralement en pointe atténuée (fig. 1, J) et le noyau de forme ovale peut atteindre $75 \mu$ dans son plus grand axe. Sa position dans le deutomérite est variable : tantôt il est situé au sommet de celui-ci, au voisinage du septum, tantôt au milieu, parfois enfin près de l'amincissement postérieur.

L'entocyte du deutomérite est plus dense que celui du protomérite.

Les sporadins sont du type Stylocéphalide habituel.

\section{CYSTOCEPHALUS ALBRECHTI n. sp.}

Une douzaine de préparations de frottis du contenu intestinal de Trachyderma hispida Forsk. nous permettent de mettre en évidence cette espèce inédite.

Les plus jeunes céphalins (fig. 2, A, B) observés mesuraient un peu plus de $100 \mu$. L'épimérite est alors encore coiffé de débris de l'épithélium intestinal de l'hôte reconnaissables à leurs noyaux (fig. 2, C, D), mais chez certains individus on voit l'épimérite qui est constitué par un volumineux bouton ponctué (fig. 2, E).

La forme de la Grégarine est ovoïde. Il n'y a pour ainsi dire pas de constriction au septum et le noyau sphérique mesure de $20 \mu$ à $30 \mu$ de diamètre.

D'autres céphalins plus âgés atteignent ou dépassent $150 \mu$ (fig. 2, F, G). L'épimérite maintenant dégagé de l'épithélium intestinal de l'hôte est tout à fait étudiable.

Il se présente comme un gros bouton plus ou moins aplati faisant suite au protomérite qui présente une sorte de bourrelet (fig. 2, H-J). Parfois on observe à l'immersion une série de petits lobes à la base du bouton (au nombre d'une quinzaine environ par face visible de céphalin) (fig. 2, H, I).

Ces céphalins ont un épicyte assez épais, l'entocyte est peu granuleux. Le noyau toujours sphérique peut atteindre $40 \mu$ de diamètre.

Les sporadins (fig. 3) sont du type Stylocéphalide habituel. Ils peuvent atteindre et même dépasser $700 \mu$.

Le protomérite y est souvent très réduit (fig. 3, B, D) et, chez certains individus, on observe la cicatrice de l'épimérite (fig. 3, C). Le noyau n'est pas toujours visible dans les gros sporadins dont l'entocyte est devenu très granuleux.

On observe chez de nombreux individus les plissements de l'épicyte de la partie postérieure du deutomérite caractéristiques du genre Cystocephalus (fig. 3, C).

Cette espèce est dédiée à notre collègue et ami M. F. O. Albrecht, Maître de Recherche au C.N.R.S. 

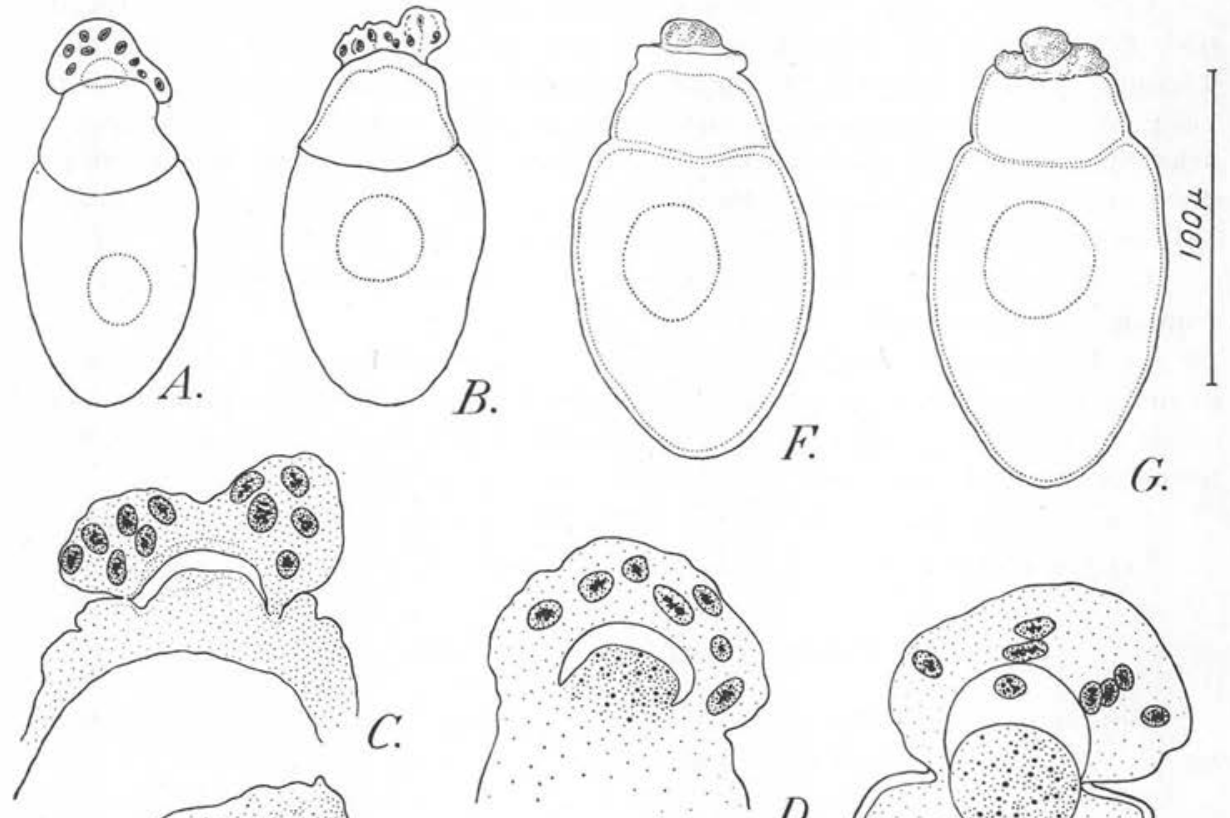

D.
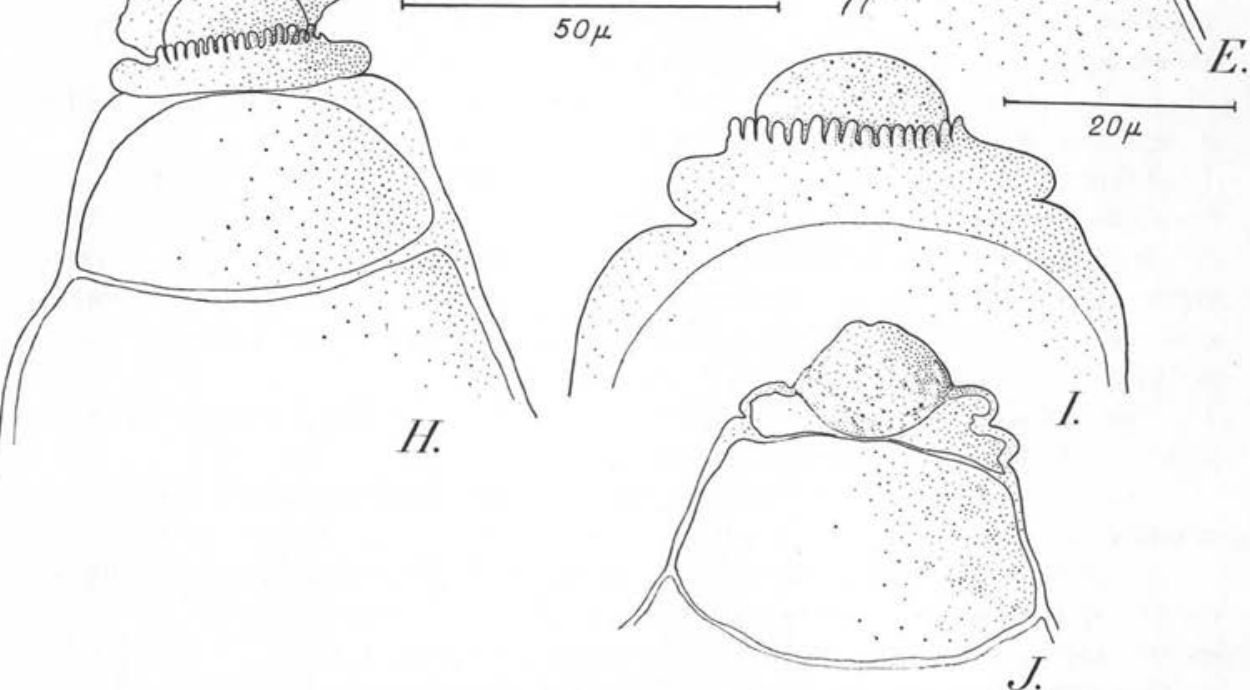

Fig. 2. - Cystocephalus albrechti n. sp. A, B, F, G, céphalins. C, D, E, épimérite avec débris de cellules épithéliales de l'intestin de l'hôte. H, I, J, détail de l'épimérite 


\section{Discussion :}

Nous avons quelque peu hésité avant de rattacher cette espèce au genre Cystocephalus car, par plusieurs caractères (présence parfois de lobes à l'épimérite, forme ovoïde des céphalins, col peu différencié, sinon absent), elle se rapprocherait du genre Lophocephalus Labbé 1899 (= Lophorhynchus A. Schneider 1882).

Cependant, dans ce dernier genre, l'épimérite est constitué par une cupule à bord crénelé, l'épicyte des trophozoïtes est strié longitudinalement et le nucléole a la forme d'un boudin contourné.

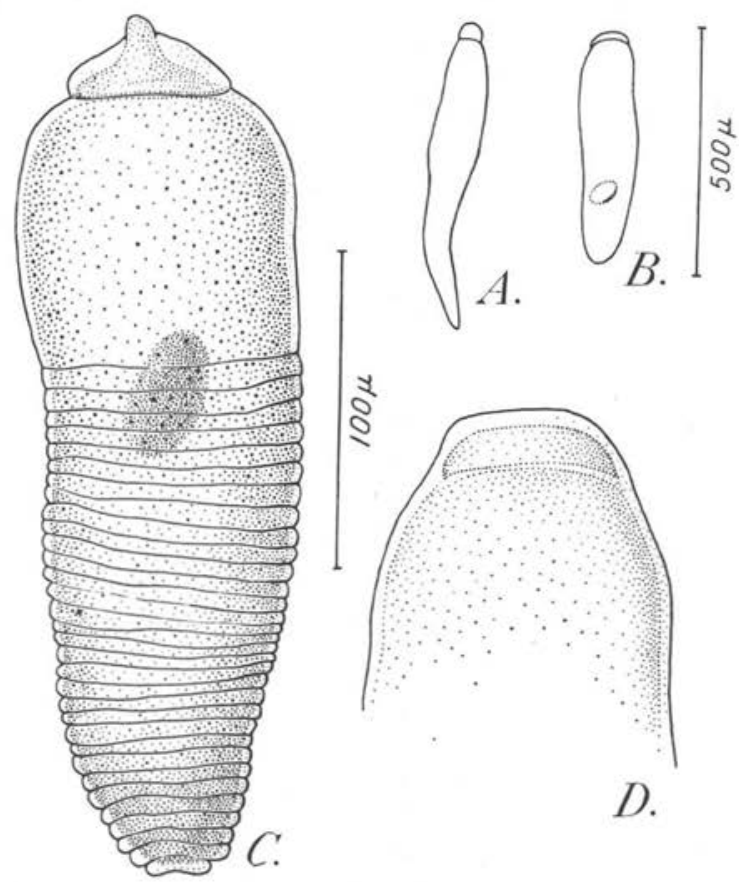

FIG. 3. - Cystocephalus albrechti n. sp. A, B, sporadins. C, sporadin avec cicatrice de l'épimérite et plissements de l'épicyte. D, détail de la région antérieure d'un sporadin

Nous pensons plus prudent, en raison de la morphologie de cette espèce (épimérite en bouton et non en cupule, sporadins à épicyte annelé) et de son hôte qui est un Pimélien, de la rapporter au genre Cystocephalus.

Notons toutefois qu'il est très possible que nous ayons affaire ici à une forme de transition entre les deux genres Cystocephalus et Lophocephalus.

Chez les Prionotheca coronata, nous avons pu mettre en évidence un genre inédit de Stylocephalidae dont voici la diagnose : 


\section{CYSTOCEPHALOIDES $n$. g.}

Genre d'Eugrégarines de la famille des Stylocephalidae caractérisé par un épimérite très particulier : tout d'abord en forme de bouton porté par un col court et entouré d'une collerette épicytaire, puis la collerette disparaît et l'épimérite prend la forme d'un bouton quadrangulaire porté par un col court. Sporadins du type Stylocéphalide habituel (1). Une seule espèce connue jusqu'ici : $C$. prionothecae n. sp. parasite de Prionotheca coronata.

Ce nouveau genre se place ainsi dans la clé des genres de Stylocephalidae, modifiée d'après celle donnée par Schneider (1886) en tenant compte de nos travaux postérieurs (Théodoridès $1955 c, 1959)$ :

I. EPIMÉRITE SANS COL BIEN DIFFÉRENCIÉ.

A. En large cupule avec une couronne de tentacules à la base: Lophocephalus (Labbé).

B. En forme de bouton parfois quadrangulaire ou tronconique : Campanacephalus Théodoridès.

C. En forme de massue quadrangulaire finement ponctuée à son apex et comprenant à l'intérieur un « îlot ponctué. Saillie du protomérite à la base du diamérite : Clavicephalus Théodoridès.

II. EPIMÉRITE A COL BIEN DIFFÉRENCIÉ.

1. Col court pas plus haut que large à la base.

A. Col cylindrique, bouton de l'épimérite globuleux ou vésiculeux sans collerette épicytaire: Cystocephalus A. Schneider.

B. Col cylindrique parfois très réduit, bouton de l'épimérite d'abord globuleux avec collerette épicytaire puis quadrangulaire sans collerette: Cystocephaloides $\mathrm{n}$. $\mathrm{g}$.

C. Col conoïde terminé par un bouton arrondi : Oocephalus (A. Schneider).

2. Col plus haut que large à la base.

A. Col terminé par un bouton trifolié ou en forme de gland : Stylocephalus Ellis.

B. Col terminé par un petit bouton sphérique Sphaerorhynchus Labbé.

Voici maintenant la description de l'espèce type de ce genre:

\section{CYSTOCEPHALOIDES PRIONOTHECAE n. sp.}

Céphalins (fig. 4 et 5) de $100 \mu$ à $400 \mu$. L'épimérite consiste dans les stades les plus jeunes en un bouton tantôt conique (fig. 4, D), tantôt globuleux (fig. 4, E), porté par un col court et entouré par une collerette finement striée.

(1) Il en sera certainement de même du kyste et des spores lorsque ces stades seront connus. 

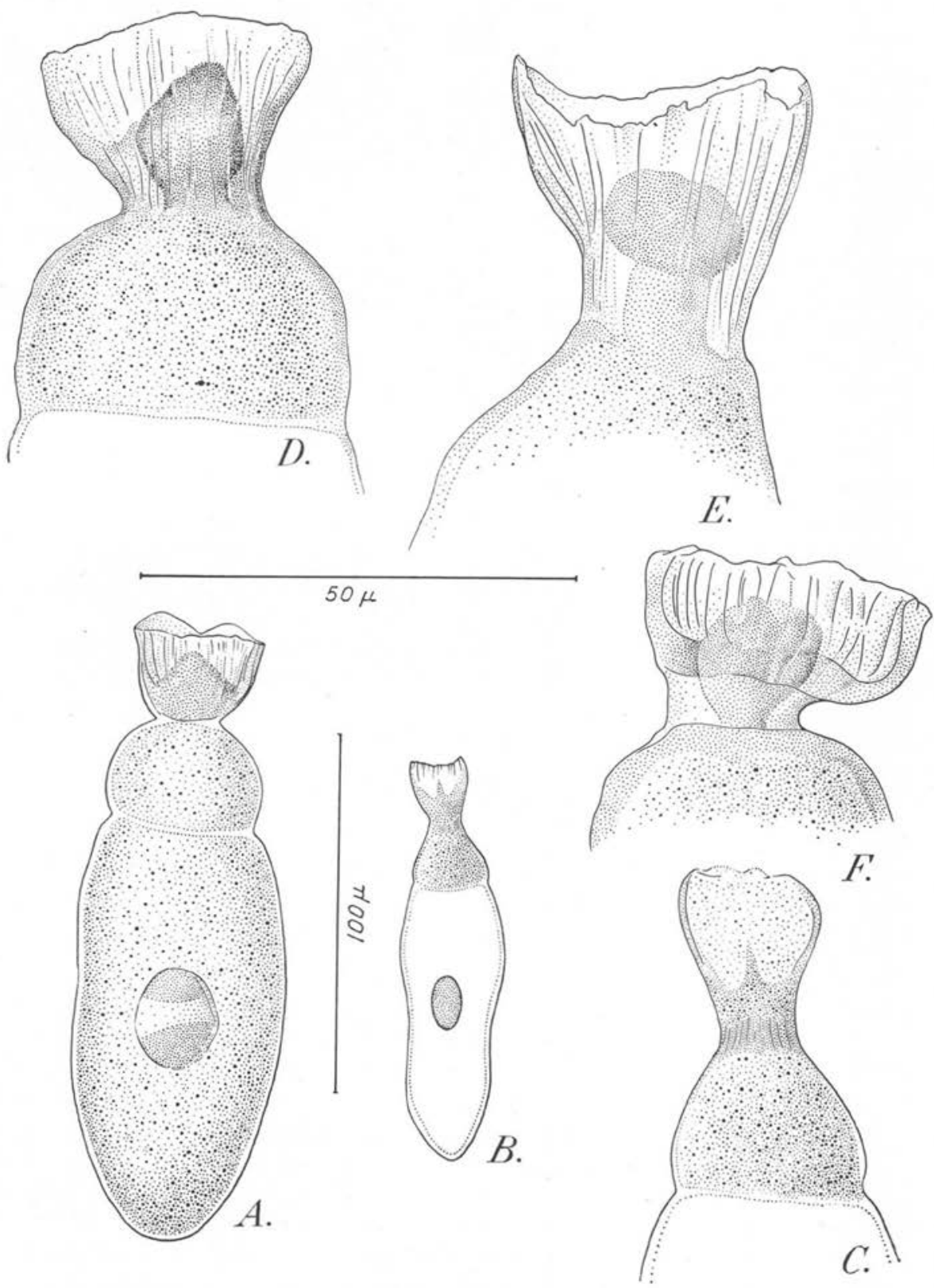

Fig. $4-$ Cystocephaloides prionothecae n. g. n. sp. A, B, céphalins. C, D, E, F, divers aspects de l'épimérite (stades avec collerette). 
Cette collerette est de nature épicytaire et sa paroi est en prolongement direct de l'épicyte du protomérite.

Toutes dimensions mises à part, cette curieuse structure (collerette + épimérite proprement dit) rappelle un manubrium de méduse entouré de son ombrelle.

Chez les jeunes céphalins, le protomérite est hémisphérique, la constriction est peu marquée au niveau du septum et le deutomérite est ovalaire avec un noyau d'environ $30 \mu$ sur $20 \mu$ (fig. 4, A).

Parfois (fig. 4, B et C), l'épimérite proprement dit est peu visible, demeurant masqué par la collerette.

Dans les céphalins plus âgés (fig. 5, A, B, C), l'épimérite s'est modifié : la collerette s'est resserrée autour du bouton épiméritique; ce stade est bien visible fig. $4, \mathrm{~F}$ et on voit sur cette figure la bordure épicytaire de la collerette de même épaisseur que celle du protomérite et le haut de cette collerette encore « ouvert » au-dessus du * manubrium » épiméritique.

Dans le stade représenté fig. 5, D, les parois de la collerette se sont resserrées encore et la forme globale de l'épimérite devient quadrangulaire, le haut de cet organite restant toujours « ouvert ».

Puis (fig. 5, H), les parois épicytaires vont se resserrer complètement et aboutir à une massue épiméritique quadrangulaire cette fois « fermée 》 à sa partie apicale et rebordée de tous côtés d'épicyte.

Comme nous l'avons signalé dans une note préliminaire (Théodoridès 1962), l'intérêt de cette transformation de l'épimérite chez $C$. prionothecae est qu'elle s'effectue chez des céphalins déjà bien différenciés. Elle n'a rien à voir avec la première différenciation structurale de cet organite qui a lieu lors du développement du céphalin dans les cellules épithéliales de l'intestin de l'hôte.

La forme des céphalins plus âgés est nettement plus allongée et le noyau mesure de 35 à $58 \mu$ environ.

Les sporadins (fig. 6, A, B) sont du type Stylocéphalide habituel et se rapprochent par leur forme de ceux du genre Cystocephalus.

Ils peuvent dépasser $500 \mu$; leur entocyte est parfois très dense et ne permet pas toujours d'observer le noyau (fig. 6, B).

Chez certains d'entre eux on observe la cicatrice de l'épimérite (fig. 6, C, D) et les plissements de l'épicyte du deutomérite (fig. 6, E) déjà signalés dans le genre Cystocephalus.

\section{ESPECES DIVERSES NON IDENTIFIABLES}

Dans ce matériel figuraient encore diverses préparations faites à partir de l'intestin moyen d'autres Ténébrionides: Opatropis hispida Brullé, Pimelia angulata Fab., Pimelia theveneti Sénac, où nous avons pu reconnaître des sporadins de Stylocéphalides.

Nous nous réservons de les étudier ultérieurement si nous obtenons du matériel complémentaire et signalons en tout cas ces Coléoptères qui sont des hôtes inédits. 


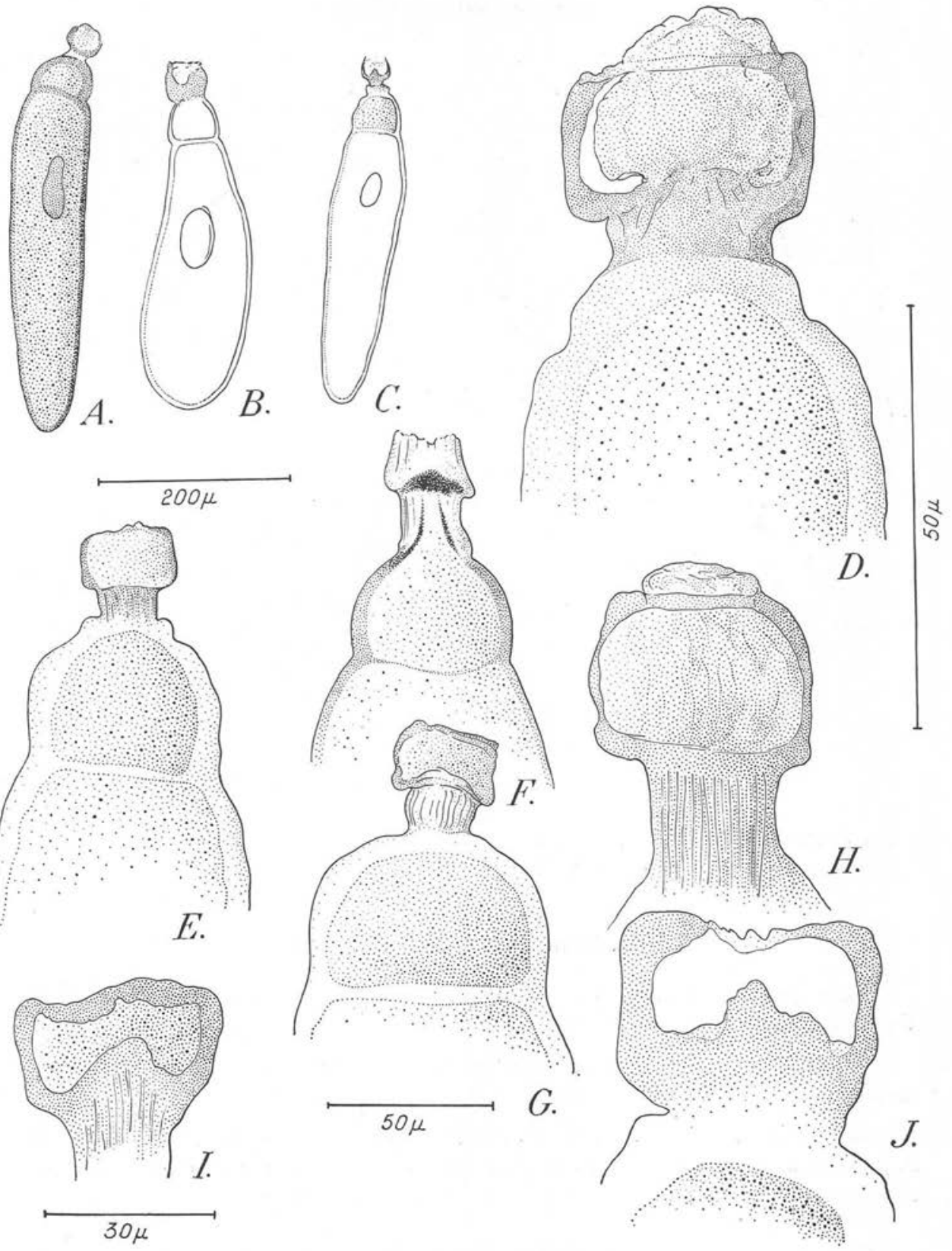

Fig. 5. - Cystocephaloides prionothecae n. g. n. sp. A, B, C, céphalins. D à I, détails de l'épimérite (après «fermeture» de la collerette) 

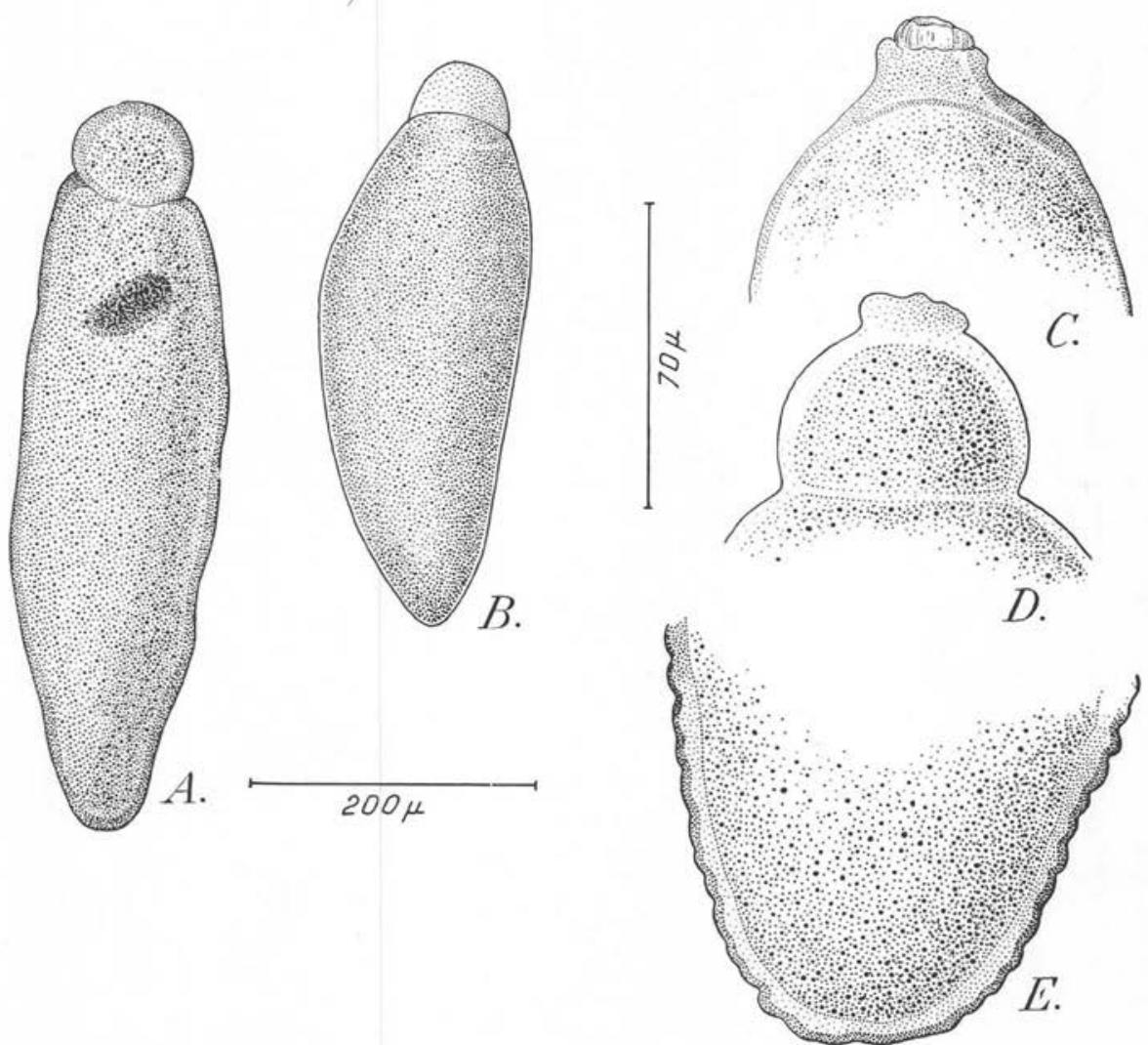

FiG. 6. - Cystocephaloides prionothecae n. g. n. sp. A, B, sporadins. C, D, cicatrices de l'épimérite. $\mathrm{E}$, plissements de l'épicyte du deutomérite

\section{Résumé et Conclusions (1)}

Cette petite collection de Grégarines Stylocéphalides parasites de Coléoptères Ténébrionides d'Egypte nous a permis :

1) De mettre en évidence ces Sporozoaires dans ce pays où, à notre connaissance, personne ne les avait encore observés.

2) De signaler des hôtes nouveaux : Blaps bifurcata (pour Stylocephalus longicollis), B. polychresta (pour $S$. eastoni), Opatropis hispida, Pimelia angulata, P. theveneti (pour des Stylocéphalides indéterminables).

(1) Au terme de cette étude, nous tenons à remercier M. P. Ardoin, qui a bien voulu déterminer les coléoptères hôtes, notre ami F. Pierre qui nous a donné dés précisions sur ces derniers et notre élève Mlle I. Desportes qui nous a aidé dans la préparation matérielle du manuscrit. 
3) De décrire un genre nouveau (Cystocephaloides n. g.) caractérisé par la morphologie de son épimérite qui subit des modifications de structure après la différenciation des céphalins.

4) De décrire une espèce nouvelle de Cystocephalus: C. albrechti n. sp.

\section{Bibliographie}

FILIPPoni (A.) (1949). - Studi sugli Stylocephalidae (Sporozoa). I. Due nuovi Stilocefalidi parassiti di Blaps gigas L. Riv. Parassitol., 10, p. 205-229.

SCHNEIDER (A.) (1886). - Grégarines nouvelles ou peu connues. Tabl. Zool., I, p. 90-103.

THÉodoridès (J.) (1955 a). - Grégarines parasites de Coléoptères Ténébrionides d'Israël. Ann. Parasit. Hum. Comp., 30, p. 161-173.

- $\quad(1955$ b). - Grégarines de Coléoptères du Maroc. Arch. Inst. Past. Maroc, 5, p. 3-14.

- $\quad(1955 c)$. - Campanacephalus villiersi n. gen. n. sp. (Eugregarina Stylocephalidae), parasite de Macropodia variolaris Ol. (Col. Tenebrionidae). Bull. I.F.A.N., XVII, sér. A, p. 813-817. (1956). - Contribution à létude des parasites et phorétiques de Coléoptères terrestres, 1 vol., 310 p., Hermann, Paris.

(1959). - Eugrégarines parasites de Coléoptères Ténébrionides de Madagascar. Mém. Inst. Sci. Mad., série A, XIII, p. 9-31.

- (1962). - Sur des modifications structurales survenant dans l'épimérite de céphalins bien différenciés de certaines Eugrégarines. C.R. Acad. Sci., 255, p. 2659-2660.

- et PIERRE (F.) (1960). - Grégarines Stylocéphalides et Coléoptères Ténébrionides. Actes $X I^{\bullet}$ Congr. Int. Entomol. (Vienne), p. 568-571.

(Laboratoire d'Evolution des êtres organisés,

Faculté des Sciences de Paris

et Laboratoire de Biologie, Université de Khartoum, Soudan) 



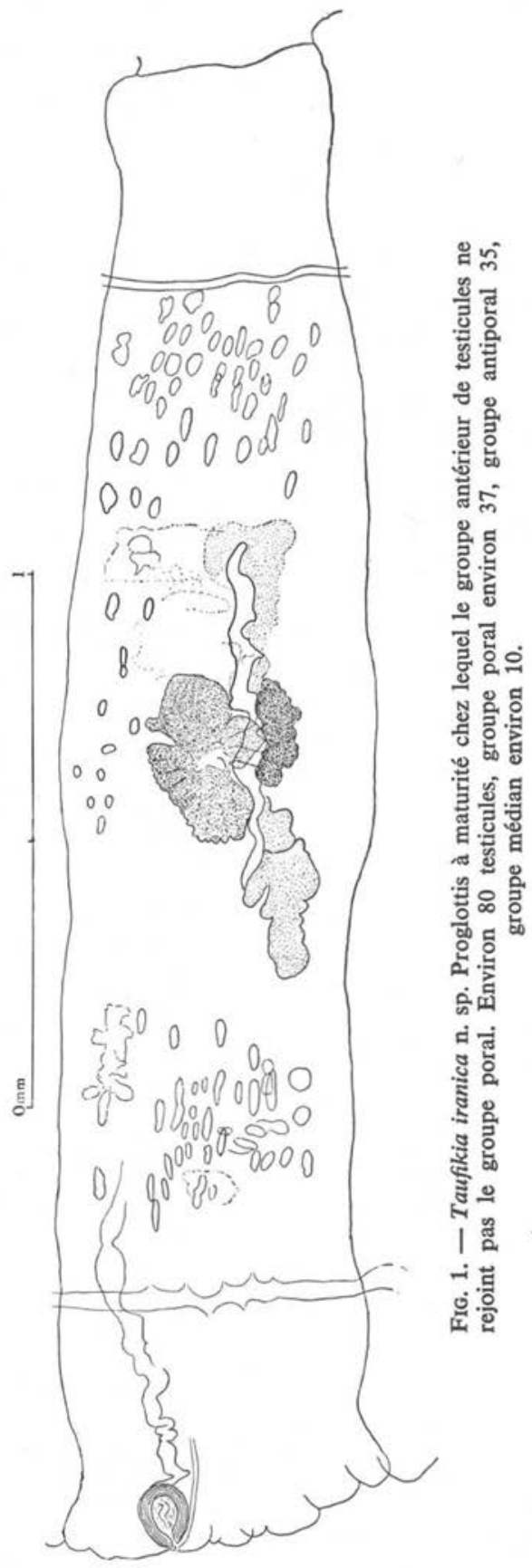

형

อै

ङ

욜

氜

छ

드

을

잉

๑

형듀

s 5

등 5 편

열을

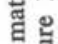

त ह

范。

웅 응

1 ํㅠㅇ

त

또물 